\title{
III. Ergänzungen und Berichtigungen (ErgVerz)
}

\begin{tabular}{|c|c|c|}
\hline Seite & Stelle & Ergänzung oder Berichtigung \\
\hline $\begin{array}{l}\text { VII } \\
\text { u. fol- } \\
\text { gende }\end{array}$ & \multirow{3}{*}{$\begin{array}{l}\text { bei } G T 44 \text { unter VIII } \\
\text { bei } G T 46 \text { Zeile } 2\end{array}$} & $\begin{array}{l}\text { werden ersetzt durch die der } 2 \text {. Lieferung beigefügten } \\
\text { Seiten VII und folgende (Abkürzungsverzeichnis usw.) }\end{array}$ \\
\hline 3 & & Es heißt: $\S 27$ \\
\hline & & Es heißt: Notarordnung \\
\hline 4 & bei $G T 50$ unter $\mathrm{Z}$ XIV & Es heißt: $\S 53$ \\
\hline 7 & $\begin{array}{l}\text { Vorbem unter } Z 1 \\
\text { Abs } 2 \text { Zeile } 3\end{array}$ & Es heißt: Abdruck (anstatt: Ausdruck) \\
\hline 10 & $\S 7$ II GBO Zeile 1 & Es heißt: Ist das Recht eine Dienstbarkeit \\
\hline 14 & $\begin{array}{l}\text { § } 35 \text { GBO Abs } 1 \text { Zeile } 2 \\
\text { Abs } 2 \text { Zeile } 1 \\
\text { § } 36 \text { I GBO Zeile } 4\end{array}$ & $\begin{aligned} \text { Es heißt: } & \text { Erbfolge } \\
& \text { fortgesetzten } \\
& \text { Eigentumsübergangs }\end{aligned}$ \\
\hline 18 & $\S 58$ II GBO Zeile 2 & Es heißt: aufzunehmen (anstatt: anzunehmen) \\
\hline 20 & $\S 77 \mathrm{GBO}$ & Es heißt: Beschwerdegerichts \\
\hline 22 & $\begin{array}{l}\S 87 \mathrm{GBO} \text { unter } \\
\text { c) Zeile } 1\end{array}$ & Es heißt: versehenden \\
\hline 33 & $\begin{array}{l}\text { § } 3 \text { II GBVerfgg } \\
\text { Zeile } 3\end{array}$ & Es heißt: von dem des sonst nach Abs. $1 \ldots$ \\
\hline 34 & $\begin{array}{l}\text { § } 6 \text { I GBVerfgg } \\
\text { Zeile } 1\end{array}$ & Es heißt: ist die Spalte 1 \\
\hline 38 & $\begin{array}{l}\text { § } 17 \text { V GBVerfgg } \\
\text { letzte Zeile }\end{array}$ & Es heißt: in \\
\hline 39 & Fußn 1 & Es ist hinzuweisen auf $\S 3 \mathrm{Bem} 258$ \\
\hline 42 & $\S 32$ GBVerfgg Zeile 4 & Es heißt: Handblatt \\
\hline 45 & $\begin{array}{l}\S 42 \text { II a GBVerfgg } \\
\text { Zeile } 5\end{array}$ & Es heißt: veräußert \\
\hline 66 & bei lfd $\mathrm{Nr} \frac{8}{\mathrm{zu} 6}$ & und 75 roter Strich (anstatt eines schwarzen Striches) \\
\hline 74 & bei lfd Nr 4 Spalte 2 & Bei 1, 2 wird die Zahl 1 rot unterstrichen \\
\hline
\end{tabular}


Ergänzungen und Berichtigungen

\begin{tabular}{|c|c|c|}
\hline Seite & Stelle & Ergänzung oder Berichtigung \\
\hline 126 & Fußn 1) & Es ist hinzuweisen auf $\S 2 \mathrm{Bem} 15$ \\
\hline 131 & $\begin{array}{l}\text { § } 31 \text { I GBOGeschO } \\
\text { Zeile } 2\end{array}$ & Es heißt: Handblatt \\
\hline 132 & $\S 36$ I GBOGeschO & $\begin{array}{l}\text { Über Änderung der Vorschrift durch Landesrecht } \\
\text { Bem zu } \$ 56\end{array}$ \\
\hline 133 & am Kopf der Seite & GT 17 (anstatt: GT 71) \\
\hline 141 & $\begin{array}{l}\S 5 \text { II AV R JM } \\
(G T 20) \text { Zeile } 1\end{array}$ & Es heißt: Nummer (anstatt: Nummern) \\
\hline 146 & $\begin{array}{l}\S 6 \text { I AV R JM } \\
(G T 23) \text { Zeile } 4\end{array}$ & $\begin{array}{l}\text { Zeile } 4 \text { heißt: Das Grundbuchamt verweist in Spalte } 10 \\
\text { der alten Durchschrift auf diese. }\end{array}$ \\
\hline 147 & $\begin{array}{l}\S 10 \text { AV R JM }(G T 23) \\
\text { Zeile } 2\end{array}$ & Es heißt: ist (anstatt: sind) \\
\hline 148 & $\begin{array}{l}\text { Anlage } 1(G T 24) \\
\text { in Mitte der Seite }\end{array}$ & $\begin{array}{l}\text { Es heißt unter } Z \text { 2: Vervollständigung } \\
\text { Es heißt vor } Z \text { 3: Die (Geschäftsstelle usw) }\end{array}$ \\
\hline 152 & $\mathrm{AV} \mathrm{RJM}(G T 26)$ & $\begin{array}{l}\text { Die AV R JM zitiert die 2. Auflage des Rösch-Kurandt. } \\
\text { In der nunmehrigen 3. Auflage (LitVerz) ist die } \\
\text { Seitenzahl die gleiche }\end{array}$ \\
\hline 165 & $\begin{array}{l}\S 3 \text { II WEGVerfgg } \\
(G T 33) \text { Zeile } 3\end{array}$ & Es heißt in Klammer richtig: ( $\$ 12$ WEG) \\
\hline 169 & $\begin{array}{l}\text { Anlage } 1(G T 34) \\
\text { vorletzte Zeile }\end{array}$ & $\begin{array}{l}\text { Roter Strich unter (Wohnungs- und Teileigentums- } \\
\text { grundbuch) }\end{array}$ \\
\hline 170 & $\begin{array}{l}\text { Spalte } 3 \text { Zeile } 5 \text { von } \\
\text { unten }\end{array}$ & $\begin{array}{l}\text { Es heißt: Sondereigentumsrechte (anstatt: Sonder- } \\
\text { eigentumsrecht) }\end{array}$ \\
\hline 191 & $\begin{array}{l}\S 21 \text { III Abs } 2 \text { AktO } \\
(G T 4 I) \text { Zeile } 3\end{array}$ & $\S 9 \mathrm{GBO}$ aF entspricht $\S 10 \mathrm{GBO} \mathrm{nF}$ \\
\hline 194 & $\begin{array}{l}\text { Anlage II }(G T 42) \\
\text { unter Bem } 4 \text { Zeile } 3\end{array}$ & $\begin{array}{l}\text { Es heißt im heutigen Sprachgebrauch: Grunderwerb- } \\
\text { steuerbescheinigung }\end{array}$ \\
\hline 198 & $\begin{array}{l}\text { EntlVerfgg }(G T 44) \\
\text { §1 II Zeile 2, } \\
\S 2 \text { IV Zeile } 3\end{array}$ & $\begin{array}{l}\text { Es heißt: Beamten (anstatt: Beamte) } \\
\text { Das Komma am Schluß der Zeile nach dem Wort „,so“ } \\
\text { entfällt }\end{array}$ \\
\hline 206 & $\S 32$ FGG Zeile 5 & Es heißt: Rechtsgeschäfte (anstatt: Rechtsgeschäft) \\
\hline 211 & $\begin{array}{l}\S 1 \text { I bad AG GBO } \\
(G T 48) \text { Zeile } 2 \\
\text { FuBn *) vorletzte } \\
\text { Zeile }\end{array}$ & $\begin{array}{l}\text { An Stelle der } \S \S 83,85,86 \mathrm{GBO} \text { aF ist } \S 117 \mathrm{GBO} \mathrm{nF} \\
\text { getreten. } \\
\text { Einschaltung nach }(296): / 27 . \text { März } 1931 \mathrm{GVBl} 77\end{array}$ \\
\hline 213 & $\begin{array}{l}\S 12 \text { I bad AG GBO } \\
(G T 48) \text { Zeile } 1\end{array}$ & Es heißt: Grundstücke (anstatt: Grundbücher) \\
\hline
\end{tabular}


Ergänzungen und Berichtigungen

\begin{tabular}{|c|c|c|}
\hline Seite & Stelle & Ergänzung oder Berichtigung \\
\hline 215 & $\begin{array}{l}\S 23 \text { II bad AG GBO } \\
(G T 48) \text { Zeile } 4\end{array}$ & Es heißt: vorgelegt \\
\hline 216 & $\begin{array}{l}\S 27 \text { I bad AG GBO } \\
(G T 48) \text { Zeile } 3 \text { bis } 5\end{array}$ & $\begin{array}{l}\text { An Stelle des Art } 20 \text { des Feldbereinigungsgesetzes vom } \\
5 . \text { Mai } 1856 \text { trat später } \S 32 \text { des Gesetzes über die } \\
\text { Feldbereinigung vom } 27 \text {. März } 1931 \text { bad GVBl 27; } \\
\text { nunmehr ist auf die Flurbereinigung hinzuweisen, } \\
\text { E } 211 f \text {. }\end{array}$ \\
\hline 217 & Fußn*) am Schluß & $\begin{array}{l}\text { Es ist anzufügen: letztmals 20. August } 1932 \text { bad } \\
\text { GVBl 228. }\end{array}$ \\
\hline 219 & $\begin{array}{l}\text { Überschrift zu } \$ 2 \\
\text { bad GBO VollzVO } \\
(G T 50)\end{array}$ & Es heißt: Dienstaufsicht \\
\hline 220 & $\mathrm{FuBn} *)$ & $\begin{array}{l}\text { Zum Ausdruck ,"Ratschreiber" und ,Ratsschreiber““ } \\
\text { S } 557 \mathrm{FuBn} \text { () }\end{array}$ \\
\hline 222 & $\begin{array}{l}\S 17 \text { bad GBO Vollz- } \\
\text { VO }(G T 50) \text { Zeile } 2\end{array}$ & Dem $\S 8 \mathrm{GBO}$ aF entspricht $\S 9 \mathrm{GBO} \mathrm{nF}$. \\
\hline 223 & $\begin{array}{l}\S 22 \text { bad GBO Vollz- } \\
\text { VO }(G T 50) \text { Zeile } 3 \\
\S 23 \text { I aaO Zeile } 2 \\
\S 25 \text { II aaO Zeile } 1\end{array}$ & $\begin{array}{l}\text { Dem } \S 45 \mathrm{GBO} \text { aF entspricht } \S 44 \mathrm{GBO} \mathrm{nF} . \\
\text { Es heißt: Grundbuch (anstatt: Grundstück) } \\
\text { Dem } 99 \mathrm{GBO} \text { aF entspricht } \S 10 \mathrm{GBO} \mathrm{nF} \text {. }\end{array}$ \\
\hline 225 & $\begin{array}{l}\S 34 \text { IV } Z 2 \text { bad AG } \\
\text { GBO }(G T 50) \text { Zeile } 2\end{array}$ & Es heißt: Nummer (anstatt: Nummern) \\
\hline 231 & $\begin{array}{l}\text { Art } 10 \text { I wü AG BGB } \\
\quad(G T 51)\end{array}$ & Es heißt: Amtsgerichtsbezirk \\
\hline 234 & $\begin{array}{l}\text { Art } 29 \text { I wü AG BGB } \\
(G T 5 I) \text { Zeile } 1\end{array}$ & Es heißt: Einem (anwesenden Beteiligten) \\
\hline 235 & $\begin{array}{l}\text { Art } 35 \text { II wü AG BGB } \\
(G T 51) \text { Zeile } 2\end{array}$ & Es heißt: nächsthöhere \\
\hline 244 & $\begin{array}{l}\S 11 \text { II wii GBVO } \\
(G T 53) \text { Zeile } 4\end{array}$ & Es heißt: Dienstaufsichtsbehörden \\
\hline 248 & $\begin{array}{l}\S 27 \text { II wü GBVO } \\
(G T 53) \text { Zeile } 3\end{array}$ & 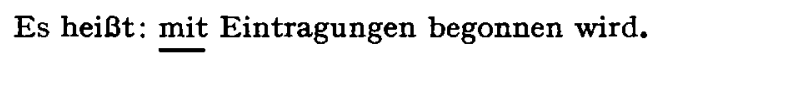 \\
\hline 252 & $\begin{array}{l}\S 39 \text { VI wü GBVO } \\
(G T 53) \text { Zeile } 3\end{array}$ & Es heißt: eingetretener \\
\hline 253 & Fußn 3) & Es wird verwiesen auf S 247 FuBn 1 (nicht 2) \\
\hline 254 & $\begin{array}{l}\S 43 \text { III wii GBVO } \\
(G T 53) \text { Zeile } 8\end{array}$ & Es heißt: ist (Verweisung usw) \\
\hline 257 & $\begin{array}{l}\S 51 \text { III wii GBVO } \\
(G T 53) \text { Zeile } 2\end{array}$ & $\begin{array}{l}\text { Es heißt: in einem besonders hierfür bestimmten } \\
\text { Umschlagbogen }\end{array}$ \\
\hline
\end{tabular}


Ergänzungen und Berichtigungen

\begin{tabular}{|c|c|c|}
\hline Seite & Stelle & Ergänzung oder Berichtigung \\
\hline 261 & $\begin{array}{l}\S 76^{\circ} \text { wï GBVO } \\
(G T 53) \text { Zeile } 1\end{array}$ & Es heißt: (statutarische) \\
\hline 262 & $\begin{array}{l}\S 81 \text { I wü GBVO } \\
(G T 53) \text { Zeile } 4\end{array}$ & Es heißt: umgeschrieben (anstatt: umschrieben) \\
\hline 264 & $\begin{array}{l}\S 96 \text { III wü GBVO } \\
(G T 53)\end{array}$ & Es heißt: Erscheinen (anstatt: Erbschein) \\
\hline 265 & $\begin{array}{l}\S 101 \text { I wü GBVO } \\
(G T 53) \text { Zeile } 1\end{array}$ & $\begin{array}{l}\text { Am Schluß der Zeile ein Punkt; es heißt daher: durch } \\
\text { die Post. }\end{array}$ \\
\hline 266 & $\begin{array}{l}\text { Karteiblatt ( } G T 54) \\
\text { in Spalte } 1\end{array}$ & Es heißt: $1,5 \mathrm{~cm}$ \\
\hline 268 & $\begin{array}{l}\S 3 \text { I Z } 9 \text { VO }(G T 56) \\
\text { Zeile } 1\end{array}$ & Es heißt: Abnahme (anstatt: Annahme) \\
\hline 279 & $\begin{array}{l}E 1 \text { Abs } 2 \text { Zeile } 4 \\
\text { letzter Absatz } \\
\text { Zeile } 2 \text { von unten }\end{array}$ & $\begin{array}{l}\text { Es heißt: Art (anstatt: } \S \text { ) } \\
\text { DRiZ 1935, } 251 \text { (anstatt: 151) }\end{array}$ \\
\hline 280 & $E 2$ Abs 1 Zeile 2 & Es heißt: vom 5. August 1935 (anstatt 15. August 1935) \\
\hline 281 & $\begin{array}{l}E 5 \text { in } \S 11 \text { des } \\
\text { 3. Ủberleitungs } \\
\text { Zeile } 2 \\
\text { in } \S 13 \mathrm{I} \text { aaO Zeile } 2\end{array}$ & Es heißt: Grundgesetzes (anstatt: Grundbuchgesetzes) \\
\hline 283 & $E 6$ & Bezüglich Bayern $\S 2$ Bem 101 \\
\hline 285 & Zeile 20 von unten & Es heißt: Reichsrats \\
\hline 286 & $\begin{array}{l}\text { Zeile } 20 \text { von oben } \\
\text { Zeile } 6 \text { von unten } \\
\text { lezte Zeile }\end{array}$ & $\begin{array}{c}\text { Es heißt: festgestellt (anstatt: festgesetzt) } \\
\text { Z JA brZ (anstatt: bzZ) } \\
\text { letztere (anstatt: letzterer) }\end{array}$ \\
\hline 287 & Zeile 4 von oben & Es heißt: Anwendbarkeit (anstatt: Anwendung) \\
\hline 288 & Zeile 14 von unten & Es heißt: Konsensprinzips \\
\hline 289 & $\begin{array}{l}\text { Zeile } 22 \text { von unten } \\
\text { Zeile } 5 \text { von unten }\end{array}$ & $\begin{array}{c}\text { Es heißt: hierin (wohl besser usw) } \\
\text { materiellrechtlich }\end{array}$ \\
\hline 290 & Zeile 14 von oben & Es heißt: rechtsbegründende \\
\hline 292 & Zeile 2 von oben & Es heißt: materiellrechtlich \\
\hline 294 & $E 18$ Abs 1 Zeile 2 & Es heißt: zwischen dem materiellen (usw) \\
\hline 295 & $E 19$ Abs 2 Zeile 2 & $\begin{array}{l}\text { Es heißt: }(v g l E 27,29,49) \text {; der Hinweis } E 48 f \text {. } \\
\text { entfällt. }\end{array}$ \\
\hline 300 & $E 25$ Abs 1 Zeile 1 & Es heißt: in (zeitlicher Übersicht usw) \\
\hline 301 & letzte Zeile & Es heißt: 1938 \\
\hline
\end{tabular}


Ergänzungen und Berichtigungen

\begin{tabular}{|c|c|c|}
\hline Seite & Stelle & Ergänzung oder Berichtigung \\
\hline 302 & $\begin{array}{l}\text { bei AV R JM v } \\
\text { 5.6. } 1937 \text { Zeile } 3\end{array}$ & Es heiBt: D J 322 (anstatt: 1322) \\
\hline 304 & $\begin{array}{l}\text { bei AV RJM v } \\
\text { 29. 12. } 1939 \text { Zeile } 2\end{array}$ & Es heißt: im \\
\hline 313 & $\begin{array}{l}E 28 \text { Zeile } 8 \\
\text { Schiffsregister Abs } 3 \\
\text { Zeile } 7 \\
\text { E } 29 \text { Zeile } 6\end{array}$ & $\begin{array}{l}\text { Bei Steiner-Riedel ZVG in 7. Auflage (1955) S } 65 \\
\text { Es heißt: näheren (Regelung usw) } \\
\text { Bei Steiner-Riedel ZVG in 7. Auflage (1955) S } 67\end{array}$ \\
\hline 314 & $\begin{array}{l}\text { E } 30 \text { Abs } 4 \text { Zeile } 1 \\
\text { Abs } 5 \text { Zeile } 6 \\
\text { Zeile } 11\end{array}$ & $\begin{array}{l}\text { Am Schluß der Zeile ein Komma, also: Rechtsanwen- } \\
\text { dung, } \\
\text { Es heißt: unserem (ganzen Rechtssystem usw) } \\
\text { Rechtsprechung }\end{array}$ \\
\hline 316 & Zeile 7 von unten & Es heißt: Landesschätzungsbeiräte \\
\hline 317 & Zeile 3 bis 5 & $\begin{array}{l}\text { Die Bek des bay JustMin v. 15. } 9.1952(G T 29) \text { bezieht } \\
\text { sich auf das Liegenschaftskataster }\end{array}$ \\
\hline 318 & Zeile 4 von unten & Es heißt: jetzt \\
\hline 319 & $\begin{array}{l}\text { E } 36 \\
\text { Zeile } 18 \text { von unten } \\
\text { letzte Zeile von unten }\end{array}$ & $\begin{array}{l}\text { Es wird auf } \S 2 \text { Bem } 99 \text { hingewiesen. } \\
\text { Es heißt: Beleihungswesens } \\
\text { Es heißt: } \S 6 \text { BodSchätzG }\end{array}$ \\
\hline 325 & E 43 Abs 7 Zeile 1 & Es heißt: Bezüglich \\
\hline 326 & Zeile 16 von unten & Es heißt: Bezeichnungen \\
\hline 329 & E 48 Abs 2 Zeile 1 & Es heißt: das (Liegenschaftskataster) \\
\hline 331 & Zeile 4 von unten & Es heißt: so (anstatt: wo) \\
\hline 334 & $\begin{array}{l}\text { bei } E 57 \\
\text { bei } E 58\end{array}$ & $\begin{array}{l}\text { Bei Überschrift Bezeichnung: C } \\
\text { Bei Ủberschrift Bezeichnung: D }\end{array}$ \\
\hline 335 & bei $E 59$ letzte Zeile & Es heißt: Obststräuchern \\
\hline 338 & $E 62$ Zeile 4 & Es heißt: Schätzungsplan üblich (Komma entfällt) \\
\hline 339 & Zeile 19 von unten & Es heißt: eingetragen (anstatt: einzutragen) \\
\hline 340 & Zeile 3 von oben & Es heißt: Eintragungen \\
\hline 342 & Zeile 11 von unten & Es heißt: eine der Anzahl usw \\
\hline 343 & Zeile 7 von unten & Es heißt: Kriegel \\
\hline 346 & Anlage 3 & $\begin{array}{l}\text { Es heißt bei Utberschrift: Vergleichendes Nummern- } \\
\text { verzeichnis }\end{array}$ \\
\hline 348 & $\begin{array}{l}E 73 \text { Abs } 1 \text { Zeile } 3 \\
\text { Zeile } 17 \text { von unten }\end{array}$ & $\begin{array}{l}\text { Es heißt: einzelne (Karte usw) } \\
\text { Es heißt: Gebäudebesitzung }\end{array}$ \\
\hline
\end{tabular}


Ergänzungen und Berichtigungen

\begin{tabular}{|c|c|c|}
\hline Seite & Stelle & Ergänzung oder Berichtigung \\
\hline 352 & Zeile 10 von oben & Es heißt: Devisenrecht \\
\hline 354 & $E 79$ Zeile 9 & Es heißt: $\S 1006 \mathrm{BGB}$ \\
\hline 356 & Zeile 22 von unten & Es heißt: Bundesgebiet \\
\hline 360 & Zeile 13 von unten & Es heißt: RGR BGB $\S 903$ Bem 3 \\
\hline 363 & E 91 Abs 3 Zeile 4 & Es heißt: Rechts, das (usw); Komma (anstatt Punkt) \\
\hline 368 & Zeile 11 & $\begin{array}{l}\text { Es heißt: Haus nicht oder in einer Form usw (das Wort } \\
\text { nicht nach oder entfällt) }\end{array}$ \\
\hline 370 & Zeile 14 von unten & Es heißt: ausreichende (Gründe) \\
\hline 371 & $\begin{array}{l}\text { Zeile } 5 \text { von unten } \\
\text { Zeile } 3 \text { von unten }\end{array}$ & $\begin{aligned} \text { Es heiBt: } & \text { Rohloff (anstatt: Rohlaff) } \\
& \text { Ähnlichkeit }\end{aligned}$ \\
\hline 374 & $\begin{array}{l}\text { Abs } 2 \text { Zeile } 4 \\
\text { Zeile } 27 \text { von unten } \\
\text { Zeile } 17 \text { von unten }\end{array}$ & $\begin{array}{l}\text { Es heißt: } \\
\text { vorgesehen ist (anstatt: sei) } \\
\text { Altenteils }\end{array}$ \\
\hline 375 & Zeile 5 von oben & Es heißt: Übernehmers \\
\hline 376 & Zeile 15 von oben & Es heißt bei der Verweisung: $(E$ 119) anstatt Bem $5 \mathrm{G}$ \\
\hline 377 & E 111 Abs 2 Zeile 4 & Es heißt: muß, da (usw); (Komma anstatt Punkt) \\
\hline 378 & Zeile 6 von oben & Thieme GBO S 315 bezieht sich auf die 3. Aufl (LitVerz) \\
\hline 379 & Zeile 21 von unten & $\begin{array}{l}\text { Es heißt: Für den Übergang sind allein die materiell- } \\
\text { rechtlichen Vorschriften des Sachenrechts des BGB } \\
\text { maßgebend; ...... } \\
\text { Es heißt: Rechtsgeschäfte (anstatt: Rechtsgeschäft) }\end{array}$ \\
\hline 380 & $\begin{array}{l}\text { E } 116 \\
\text { Zeile } 24 \text { von unten } \\
\text { Zeile } 17,18 \text { von } \\
\text { unten } \\
\text { Zeile } 3 \text { von unten }\end{array}$ & $\begin{array}{l}\text { Es heißt: } \S \S 896,1116 \text { usw (Komma) } \\
\text { Bei DRspr entfällt die Verweisung auf I (154) } 2 \text { Rücks } \\
\text { DRspr I (154) } 2 \text { Rücks entspricht nunmehr DRspr I } 3 \\
\text { der Lieferung } 2 / 56\end{array}$ \\
\hline 381 & $\begin{array}{l}\text { Zeile } 26 \text { von unten } \\
\text { Zeile } 14 \text { von unten }\end{array}$ & $\begin{array}{l}\text { Es heißt: die (Eintragung im Grundbuch) } \\
\text { der (durch Rangvorbehalt usw) }\end{array}$ \\
\hline 382 & $\begin{array}{l}\text { E } 118 \text { lezte Zeile } \\
\text { Zeile } 4 \text { von unten }\end{array}$ & $\begin{aligned} \text { Es heißt: Eigentümers } & \\
& \text { dingliche (Unterwerfungsklausel usw) }\end{aligned}$ \\
\hline 383 & Zeile 9 von oben & Bei Steiner-Riedel ZVG in 7. Auflage (1955) S 33 \\
\hline 385 & Zeile 7 von oben & Es heißt: Gebäude \\
\hline 387 & Zeile 6 von unten & Es heißt: Zweifeln (anstatt: Zweifel) \\
\hline 389 & $\begin{array}{l}\text { Zeile } 5 \text { von oben } \\
\text { Zeile } 6 \text { von unten }\end{array}$ & $\begin{array}{l}\text { DRspr I (154) } 3 \text { Rücks entspricht nunmehr DRspr I } \\
\quad(154) 4 \text { Rücks bei Lieferung } 2 / 56 \\
\text { In Übersicht unter } 3 \text { b heißt es: Dauerwohnrechts }\end{array}$ \\
\hline
\end{tabular}


Ergänzungen und Berichtigungen

\begin{tabular}{|c|c|c|}
\hline Seite & Stelle & Ergänzung oder Berichtigung \\
\hline 390 & $E 135$ zum Absatz 5 & $\begin{array}{l}\text { Es ist darauf hinzuweisen, daß das wïba Ges Nr } 275 \\
\text { nebst den VOen Nr 291, } 292 \text { durch das Ges v } 16.2 . \\
1953 \text { bawü GVBl } 1953,9=\mathrm{SaBl} 194 \text { aufgehoben } \\
\text { wurde; es findet nur noch auf Miteigentum und Erb- } \\
\text { baurecht, die am } 1.1 .1953 \text { bestanden, weiter An- } \\
\text { wendung. } \\
\text { Es heißt: } \S 48 \text { II } 2 \text { WEG erhielt ... eine neue Fassung. } \\
\text { Es ist auch darauf hinzuweisen, daB das Saarland } \\
\text { ein WEG vom } 13.1 .1952 \text { saarl ABl } 686 \text { hat, das } \\
\text { fast den gleichen Wortlaut wie das WEG der BRD } \\
\text { hat. }\end{array}$ \\
\hline 391 & $\begin{array}{l}\text { E } 137 \\
\text { Bei E } 137 \text { Absatz } 3 \\
\text { Zeile } 1\end{array}$ & $\begin{array}{l}\text { Palandt BGB und Weitnauer-Wirths WEG sind } \\
\text { inzwischen in Neuauflage erschienen, dazu Lit Verz, } \\
\text { Es heiBt bei Brand-Schnitzler: } \S 167\end{array}$ \\
\hline 392 & Zeile 14 von oben & Es heißt: Gebäude (eingeräumt usw) \\
\hline 394 & Zeile 3 von unten & Es heißt: Zwangsvollstreckung \\
\hline 997 & Zeile 11 von unten & Es heißt: am (Grunstück usw) \\
\hline 402 & $E 156$ & $\begin{array}{l}\text { Zum LwVG ist auch der Kommentar von Pritsch er- } \\
\text { schienen, vgl LitVerz. }\end{array}$ \\
\hline 404 & $\begin{array}{l}\text { Zeile } 15 \text { von oben } \\
\text { Zeile } 21 \text { von unten } \\
\text { Zeile } 8 \text { von unten }\end{array}$ & $\begin{array}{l}\text { Es heißt: zum Wert des Grundstücks steht; } \\
\text { Es entfällt das Wort: die } \\
\text { Es heißt: privatrechtlich (Berechtigten usw) }\end{array}$ \\
\hline 406 & $\begin{array}{l}\text { Zeile } 5 \text { von oben } \\
\text { Zeile } 19 \text { von unten }\end{array}$ & $\begin{array}{l}\text { Es heißt: BGH } \\
\text { Es heiBt bei DRspr II : (284) anstatt (294) }\end{array}$ \\
\hline 408 & Zeile 16 von unten & Es heiBt: der (Rentenbank-Kreditanstalt usw) \\
\hline 411 & $\begin{array}{l}\text { Zeile } 14 \text { von oben } \\
\text { Zeile } 10 \text { von unten } \\
\text { Zeile } 5 \text { von unten }\end{array}$ & $\begin{array}{l}\text { Es heißt: festgesetzte (steuerliche Wert) } \\
\text { waren, findet (Komma) } \\
\text { verringert }\end{array}$ \\
\hline 413 & Zeile 17,18 von unten & Es heißt: übergehen (anstatt: übergeben) \\
\hline 414 & letzte Zeile & Es heißt: Höver (anstatt: Hörer) \\
\hline 415 & Übersicht unter $7 \mathrm{~b}$ & Es heißt: Rechte \\
\hline 416 & Zeile 13 von oben & Es heißt bei Bodenreform: (E 205 bis 210) \\
\hline 417 & $\begin{array}{r}\S 1 \text { I WSG Zeile } 4 \\
\text { Zeile } 6\end{array}$ & $\begin{aligned} & \text { Es heißt: } \text { Die (gleiche Befugnis usw) } \\
& \text { obersten (Landesbehörden usw) }\end{aligned}$ \\
\hline 418 & $\begin{array}{l}\S 5 \text { Z } 1 \text { WSG Zeile } 3 \\
\text { Zeile } 15 \text { von unten }\end{array}$ & $\begin{array}{l}\text { Es heißt: Kleinsiedlung zu Zwecken des Betriebes oder } \\
\text { der Kleinsiedlung, nicht aber (usw) } \\
\text { Überschrift: AusfVO zum WSG }\end{array}$ \\
\hline 421 & E 185 Abs 1 Zeile 1 & Es heißt: Grundsteuervergünstigung \\
\hline
\end{tabular}


Ergänzungen und Berichtigungen

\begin{tabular}{|c|c|c|}
\hline Seite & Stelle & Ergänzung oder Berichtigung \\
\hline 422 & $\begin{array}{l}\text { Zeile } 25 \text { von unten } \\
\text { Zeile } 13 \text { von unten }\end{array}$ & Es heißt: $\begin{aligned} \S & 20 \text { WGB } \\
& \text { Familienheime }\end{aligned}$ \\
\hline 426 & $E 190$ & $\begin{array}{l}\text { Das HeimstG wurde geändert durch Ges v 3. } 8.1953 \\
\text { BGBl I } 720\end{array}$ \\
\hline 427 & $\begin{array}{l}\text { Zeile } 19 \text { von oben } \\
\text { Zeile } 12 \text { von unten }\end{array}$ & $\begin{array}{l}\text { Es heißt: } \text { abgegebene } \\
\text { Landlieferungsverbänden }\end{array}$ \\
\hline 432 & $\begin{array}{l}\text { E } 195 \text { letzter Absatz } \\
\text { vor ,Eigenheime“ }\end{array}$ & $\begin{array}{l}\text { Die Einziehung der Renten erfolgt durch die Finanz- } \\
\text { ämter, die Landesbehörden sind. }\end{array}$ \\
\hline 433 & Zeile 17 von oben & Es heißt: stehen (den Betroffenen usw.) \\
\hline 439 & $\begin{array}{l}\text { Zeile } 9 \text { von oben } \\
\text { Zeile } 6 \text { von unten }\end{array}$ & $\begin{aligned} \text { Es heißt: } & \text { berichtigt } \\
& \text { WBG }\end{aligned}$ \\
\hline 440 & $\begin{array}{l}\text { Zeile } 22 \text { von unten } \\
\text { Zeile } 11 \text { von unten }\end{array}$ & $\begin{aligned} \text { Es heiBt: } & \text { BaulBesch } \mathrm{G} \\
& \text { darf (anstatt: bedarf) }\end{aligned}$ \\
\hline 441 & $E 204$ & $\begin{array}{l}\text { Zur Frage der Verfassungsmäßigkeit des gerichtlichen } \\
\text { Verfahrens BVG N JW 1956, 625. }\end{array}$ \\
\hline 447 & Zeile 5 von unten & Es heißt: es sei denn (usw) \\
\hline 448 & Zeile 20 von unten & Es heißt: zuständige (Grundbuchamt usw) \\
\hline 449 & Zeile 23 von unten & Es heißt: BGBl (anstatt: RGBl) \\
\hline 450 & $\begin{array}{l}\text { Zeile } 26 \text { von unten } \\
(E 213 \text { Abs } 4)\end{array}$ & Es heißt: Sie (hat hierbei von den Eintragungen usw) \\
\hline 451 & $E 215$ Abs 1 Zeile 3 & Es heißt: auf (anstatt: bei) \\
\hline 453 & $\begin{array}{l}\text { Zeile } 22 \text { und } 30 \text { von } \\
\text { unten }\end{array}$ & Es heißt: Amerik Zone \\
\hline 454 & Zeile 16 von oben & Es heißt: Henke-Mönch GBO \\
\hline 458 & $\begin{array}{l}E 221 \text { Abs } 1 \\
\text { Zeile } 2 \\
\text { Zeile } 3\end{array}$ & $\begin{array}{l}\text { Es heißt: Haegele GBRecht } S 4279 \\
\text { Bei Steiner-Riedel ZVG (in } 7 \text {. Aufl 1955) wird auf } \\
\text { S } 42 \text { verwiesen }\end{array}$ \\
\hline 459 & Zeile 4 von unten & Es heißt: Durchführung \\
\hline 460 & Zeile 19 von unten & Es heißt: LAG \\
\hline 461 & E 224 Abs 2 Zeile 6 & Es heißt: Lastenausgleich \\
\hline 463 & $\begin{array}{l}\text { Zeile } 9 \text { von oben } \\
\text { Zeile } 10 \text { von oben } \\
\text { Zeile } 17 \text { von unten }\end{array}$ & $\begin{aligned} \text { Es heißt: } & \text { Wiederaufbaus } \\
& \S \S 3 a, b, c \text { LSG } \\
& \text { LAG }\end{aligned}$ \\
\hline 464 & Zeile 3 von unten & Es heißt: übergegangenen \\
\hline
\end{tabular}


Ergänzungen und Berichtigungen

\begin{tabular}{|c|c|c|}
\hline Seite & Stelle & Ergänzung oder Berichtigung \\
\hline 468 & Zeile 16 von unten & Es heißt: wären, (wenn die $Z$ w erst usw) \\
\hline 470 & Übersicht unter $3 \mathrm{~b}$ & Es heißt: BEG \\
\hline 471 & Zeile 2 von oben & Es heißt: Entschädigung (für Opfer usw) \\
\hline 473 & Zeile 26 von unten & Es heißt: einem (gegen die guten Sitten usw) \\
\hline 474 & Zeile 8 von unten & Es heißt: weitgehendst \\
\hline 476 & $\begin{array}{l}\text { Zeile } 18 \text { von oben } \\
\text { Zeile } 13 \text { von unten }\end{array}$ & $\begin{aligned} \text { Es heißt: } & \text { BGHZ 11, } 16 \\
& (\text { amerik Z) anstatt (Anm 2) }\end{aligned}$ \\
\hline 479 & Zeile 12 von oben & Es heißt: rechtskräftigen \\
\hline 480 & $E 235,236$ & Es heißt: BEG (anstatt REG) \\
\hline 483 & $\begin{array}{l}\text { VO PR } 75 / 52 \text { Vor- } \\
\text { spruch Zeile } 3 \\
\S 2 \text { Zeile } 3\end{array}$ & $\begin{array}{c}\text { Es heißt: RGBl I S } 824 \\
\text { Widerruflich }\end{array}$ \\
\hline 487 & Zeile 2 von unten & Es heißt: OLG Frankfurt a M N JW 1954, 153 (H u ber) \\
\hline 489 & Zeile 13 von oben & Es heißt: ist (finden sich eingehende Ausführungen usw) \\
\hline 492 & $\begin{array}{l}\text { Abs } 4 \text { Zeile } 4 \\
\text { Zeile } 5 \\
\text { Zeile } 8 \text { von unten }\end{array}$ & $\begin{array}{l}\text { Es heißt: inwieweit } \\
\text { können, dem Enteigneten usw; (das Wort } \\
\text { "von" entfällt) } \\
\text { ausdrückliche (anstatt: ausdrücklich) }\end{array}$ \\
\hline 494 & $\begin{array}{l}\text { Zeile } 12 \text { von oben } \\
\text { Zeile } 14 \text { von oben }\end{array}$ & $\begin{array}{l}\text { Es heißt: Wiedergutmachungsangelegenheiten } \\
\text { Ausland }\end{array}$ \\
\hline 498 & Übersicht & Es heißt: 1. Allgemeines \\
\hline 503 & Zeile 21 von oben & Es heißt: Beschlagnahme \\
\hline 505 & $\begin{array}{l}\text { Zeile } 12,13 \text { von oben } \\
\text { Zeile } 10 \text { von unten } \\
\text { Zeile } 7 \text { von unten }\end{array}$ & $\begin{array}{c}\text { Es heißt: landwirtschaftlichem Vermögen, ......... } \\
\text { gärtnerischem Vermögen } \\
\text { fremdem (Grund und Boden) } \\
\text { forstwirtschaftlichen (Vermögen usw) }\end{array}$ \\
\hline 506 & Zeile 3 von unten & Es heißt: fortgesetzte \\
\hline $\begin{array}{l}507 \\
\text { und } \\
508\end{array}$ & $\begin{array}{l}\text { Zeile } 9 \\
\text { von oben } \\
\text { Zeile } 1\end{array}$ & Es heißt: insbesondere \\
\hline 507 & $E 256$ & $\begin{array}{l}\text { Zur Körperschaftssteuer ist auf die DurchführungsVO } \\
\text { v 23. 12. } 1955 \mathrm{BGBl} 1853=\text { SaBl 1956, } 38 \text { hinzu- } \\
\text { weisen }\end{array}$ \\
\hline 508 & $E 257$ & $\begin{array}{l}\text { Zur Kapitalertragssteuer ist auf die DurchführungsVO } \\
\text { in Neufassung v 25. 2. } 1956 \mathrm{BGBl} \text { I } 95=\text { SaBl } 220 \\
\text { hinzuweisen }\end{array}$ \\
\hline
\end{tabular}


Ergänzungen und Berichtigungen

\begin{tabular}{|c|c|c|}
\hline Seite & Stelle & Ergänzung oder Berichtigung \\
\hline 510 & Ziffer 4 Zeile 2 & Es heißt: Rechtsverordnung \\
\hline 511 & Zeile 18 von unten & Es heißt: begünstigten (Zwecken usw) \\
\hline 521 & Zeile 6 von unten & Es heißt: Betrachtungsweise \\
\hline 524 & $\begin{array}{l}\text { Zeile } 10 \text { von oben } \\
\text { Zeile } 12 \text { von oben }\end{array}$ & $\begin{aligned} \text { Es heißt: } & \text { Sicherung } \\
& \text { haben (anstatt: hat) }\end{aligned}$ \\
\hline 525 & letzte Zeile & Es heißt: Empfängers \\
\hline 526 & $\begin{array}{l}\text { Abschnitt über } \\
\text { Steuerschuld Zeile } 1\end{array}$ & Es heißt: Vertragsteile \\
\hline 527 & $\begin{array}{l}\text { GrEStDV } \& 2 \\
\text { I } Z 1 \mathrm{~g} \\
\text { II } Z 2\end{array}$ & $\begin{array}{l}\text { Es heißt: Handelsgesellschaft } \\
\text { fremdem (Boden usw) }\end{array}$ \\
\hline 528 & $\begin{array}{l}\text { GrEStDV } \S 4 \text { II } Z 2 \\
\text { Zeile } 2 \text { von unten }\end{array}$ & $\begin{aligned} \text { Es heißt: } & \text { Gesellschaftsanteile } \\
& \text { hat (anstatt: haben) }\end{aligned}$ \\
\hline 529 & $\begin{array}{l}\text { Muster } 1 \text { und } 2 \\
\text { unter } 2 \mathrm{~b}\end{array}$ & Es heißt: rrrundstück \\
\hline 530 & $\begin{array}{l}\text { unter } 5 \text { bei Rest- } \\
\text { kaufgeld }\end{array}$ & hypothekarisch \\
\hline 530 & Muster 2 unter $Z 6$ & Es heißt: mir (anstatt: mit) \\
\hline 532 & Zeile 3 von oben & Es heißt: Finanzamt \\
\hline 533 & $\begin{array}{l}\text { Zeile } 1 \text { von oben } \\
\text { Zeile } 21 \text { von oben }\end{array}$ & $\begin{array}{l}\text { Es heißt: Unbedenklichkeitsbescheinigung } \\
\text { Jahren (anstatt: Jahre) }\end{array}$ \\
\hline 535 & $E 275$ & $\begin{array}{l}\text { Zur bevorstehenden Neuordnung des Notarrechts } \\
\text { Schack JR 1956, } 161\end{array}$ \\
\hline 536 & $E 270$ vorletzte Zeile & Es heißt: Amtsrichter \\
\hline 538 & E 278 Zeile 3 & Es heißt: einer (Fülle usw) \\
\hline 539 & $\begin{array}{l}\S 19 \text { EntlVerfgg } \\
\text { unter b) }\end{array}$ & Es heißt: Urkunden (anstatt: Urkunde) \\
\hline 541 & $\begin{array}{l}\text { Entwurf } \$ 10 \mathrm{IV} \\
\text { Zeile } 1 \\
\text { Zeile } 2\end{array}$ & $\begin{aligned} \text { Es heißt: } & \text { stattgeben } \\
& \text { Entscheidungen }\end{aligned}$ \\
\hline 546 & $\begin{array}{l}\text { Zeile } 6 \text { von oben } \\
\text { Zeile } 23 \text { von oben }\end{array}$ & $\begin{aligned} \text { Es heiBt: } & \text { vom (Zufall usw) } \\
& \text { ein (Ehegatte beim Eintritt usw) }\end{aligned}$ \\
\hline 549 & $\begin{array}{l}\text { Zeile } 13 \text { von oben } \\
\text { Zeile } 18 \text { von unten }\end{array}$ & $\begin{aligned} \text { Es heißt: } & \text { Rechtsbeziehungen } \\
& \text { des (Ministerrats usw) }\end{aligned}$ \\
\hline 550 & Zeile 1 & Es heißt: Besorgung \\
\hline
\end{tabular}


